\title{
Assessment of the Autonomic Response to Sensory Stimulation in Autism Spectrum Disorder
}

\author{
L Cavinato $^{1}$, A Cardinaux ${ }^{2}$, W Jamal ${ }^{2}$, M Kjelgaard ${ }^{3}$, P Sinha $^{2}$, R Barbieri $^{1}$ \\ ${ }^{1}$ Politecnico di Milano, Italy \\ ${ }^{2}$ Massachusetts Institute of Technology, Massachusetts \\ ${ }^{3}$ Marymount Manhattan College, New York
}

\begin{abstract}
Defined as the ability of the nervous systems to reduce their response over repeated stimulation, habituation inflects its parameters in terms of frequency, intensity, recovery and anticipation of responses. Although its concepts have developed from the study of the Central Nervous System (CNS) in processing stimuli at the cortical level, we aim at defining habituation from an autonomic point of view, via heart rate and heart rate variability assessments. To this extent, by using a point-process approach, we devise a novel Autonomic Reactivity Function (ARF) describing the time-varying Autonomic Nervous System (ANS) response in terms of intensity and anticipation, whose reduction (or increment) over repeated stimuli can be ascribed to habituating (or sensitizating) patterns. We tested the mathematical formalization of such metrics in both neurotypical subjects and children with autism spectrum disorder. By eliciting autonomic responses via multisensory stimulation, we collected electrocardiography (ECG) signals, pulled ARFs out from them and performed the Persons coefficient between autonomic habituation metrics and participants sensory profiles and disorder severeness. Results show a relevant positive correlation with Short Sensory Profile (SSP-2) questionnaire (60\%) and with Autism Diagnostic Observation Schedule (ADOS-2) questionnaire (76\%).
\end{abstract}

\section{Introduction}

Acuity of responses in absolute terms might not be appropriate for measuring predictive abilities. Rather, these could be translated into the capacity to anticipate sensory processing in order to face the huge amount of information we are subjected to every day. In fact, a lag in processing pathways, both ascendant and descendant, would lead to an altered perception of the environment, as well as difficulties in relating and interfering with real time stimuli, thus making it hard to measure predictive behavior by looking at only autonomic responses. As a matter of fact, usually knowledge from central sensory processing is often used to estimate the impact that a stimulus has while traveling in our brain as reflected, for example, in the amplitude of its event-related potential. As this stimulus is provided several time over a temporal window, its corresponding event yields a smaller and smaller response in the brain potential. This phenomenon is called habituation [1] [2]. Habituation has been designed for event-related measurements such as EEG or EDA. Our challenge here is to define habituation by looking at autonomic changes reflected in the heartbeat variations along time, i.e. heart rate variability (HRV), by exploiting the time-varying feature from advanced models of hearbeat dynamics in order to create some even-related function that may provide the same habituation paradigm. In this context, there is evidence to suggest that children with Autism Spectrum Disorder (ASD) do not show progressive dampening of neural response to repeated standard tones or clicks [1] [3]. Perry and colleagues found that ASD participants required more trials to reach maximal habituation and suggested that ASD participants have a generalized slowing of habituation to repeated stimuli [4] [5] [6] [7]. Thus, we test and practically evaluate our metrics on ASD case study by accessing the ASD reduced habituation thesis from an autonomic point of view. According to these premises, our study aims at (a) building autonomic habituation metrics as a proxy of prediction, (b) investigating habituation reduction in ASD with multi-modal naturalistic stimuli and (c) exploring the relationship between autonomic habituation and clinical ASD parameters measured by psychological assessments of sensory, cognitive and behavioral performance.

\section{Experimental Protocol}

Twentyone neurotypical subjects (9 females) and eight ASD subjects (2 females) ranging from 7.2-12.8 years have been included in this study. All participants had a Wechsler Intelligence Scales for Children - Fifth Edition 
(WISC-V) nonverbal IQ of 85 or greater. All ASD participants met criteria for autism or autism spectrum disorder based on administration of the Autism Diagnostic Observation Schedule (ADOS-2), which describes the syndrome severeness. Moreover each participant has been subjected to a sensory profile delineation according to the Short Sensory Profile (SSP-2) questionnaire. In particular, 125 items describe the child's response to sensory experiences concerning registration, seeking, sensitivity, and avoiding sensory processing patterns [8]. The higher the scores, the higher the sensory impairment. In the recording stage, we implemented a 5-minute multisensory stimulation by repeating a video sequence including one minute of baseline video of a nonsocial scene, followed by a 30-second clip that repeated 5 times. The repeating video clips shows a person opening a jack-in-the-box, and a toy clown popping out of the box right after, representing the stimulus. ECG has been recorded for the entire duration of the experiment.

\section{Methods}

Autonomic Nervous System activity has largely been studied via sympatho-vagal balance assessment. In particular, Heart Rate Variability (HRV), i.e. the variation in either the R-R intervals (times between R-wave events) or heart rate with time around their mean [9], has been proven to be an important quantitative marker of cardiovascular regulation and a potential marker of autonomic regulation. Its significance can be appreciated in both temporal and spectral domains, where HRV can be decomposed into low frequency (LF) and high frequency (HF) components of the power spectral density: the LF-HRV reflects both sympathetic and vagal influences, whereas HF-HRV is highly correlated with vagal activity [10]. More recently, a pointprocess based model [11] has been introduced for estimating time varying LF-HRV, HF-HRV and their mutual relationship. This model allows for extraction of autonomic features in continuous time, which are summarized as follows:

$$
\left\{\begin{array}{l}
\mu_{R R}=\mu\left(H_{u_{k}}, \theta\right) \\
\sigma_{R R}=\left[\mu\left(H_{u_{k}}, \theta\right)^{3} \theta_{p+1}^{-1}\right]^{\frac{1}{2}} \\
S_{R R}(f, t)=\frac{\sigma_{R R}(t)}{1-H_{f, t}}
\end{array}\right.
$$

where $H_{f, t}=\sum_{k=1}^{r} \theta_{k}(t) \exp (-j 2 \pi j k)$, which is traded on computing LF-HRV, HF-HR and balance (i.e. $\mathrm{LF} / \mathrm{HF}$ ) features by Fourier-trasforming the vector of parameters $\theta$. According to our assumptions, the hypothetical function describing the autonomic response should be a temporal feature describing the status of the autonomic system at each point in time: it should be positive when a reaction to a stimulus is taking place and null otherwise. Exploiting the point-process model's high temporal resolution and hybrid frequency-temporal represen- tation of the autonomic regulation, we make use of the sympatho-vagal balance feature (LF/HF) for building up an Autonomic Responsivity Function (ARF). In fact, the ARF should be positive when autonomic regulation tilts towards sympathetic activity and it is negative when a perturbation of the balance towards the vagal system takes place. In this regard, such response is proportional to a positive increase in LF-HRV, determining a sympathetic prevalence, and inversely proportional to the parasympathetic system's response, i.e. the HF-HRV power congruent to the respiratory sinus arrhythmia effect. Since LF/HF represents the sympatho-vagal balance at each moment in time, such measure does not represents an absolute measure of the autonomic level, and it is further affected by individual differences, circadian rhythm and other external conditions. On the contrary, we could use a measure describing the change in autonomic levels for comparing event-wise changes over time in amplitudes and latency in building the proposed ARF configuration. First, a derivative filter is applied to the raw LF/HF feature. Then, given that the response is event-related by definition, we exploit the event timing for convenience. Specifically, from the LF/HF raw feature we consider a window in which it is physiologically likely to have a response from autonomic system is taken. In particular, the window includes 2 seconds of pre-stimulus and 5 seconds of post-stimulus, for a total of 7 second duration. An exemplary window presents a baseline wonder upon which both informative and non informative peaks pop up, whose shape, amplitude and width are not immediately accessible. In order to perform the calculation of the response magnitude, the baseline must be subtracted. To do this, a fitting curve is built and properly subtracted from the raw signal (Figure 1.a). In this way, regardless if a stimulus happens in a valley or on a hill, its value is re-scaled to a zero-based baseline. In this way, the zeros define the null response, in which no perturbation of sympathetic system is happening, while peaks are event-mediated perturbations of autonomic regulation toward sympathetic post-elicitation with respect to the vagal pre-relaxation. In other words, peaks are not only informative of the sympathetic activation, but they are rather refelecting the sympatho-vagal dynamical range, computed on a pre-post stimulus difference basis. As autonomic arousal peaks in the LF/HF raw feature are quite irregular and often overlapping, they need to be transformed, reshaped and scaled prior to building the ARF. Once peaks have been aligned along the zero-based baseline, the computation of their prominence and width as well as the delay between the stimulus and the maximum point is performed. These parameters are thus used to build a response-like curve. Specifically, since a response is intended to be a bell-shaped curve in which its magnitude is expected to grow until a maximum point and to decrease 


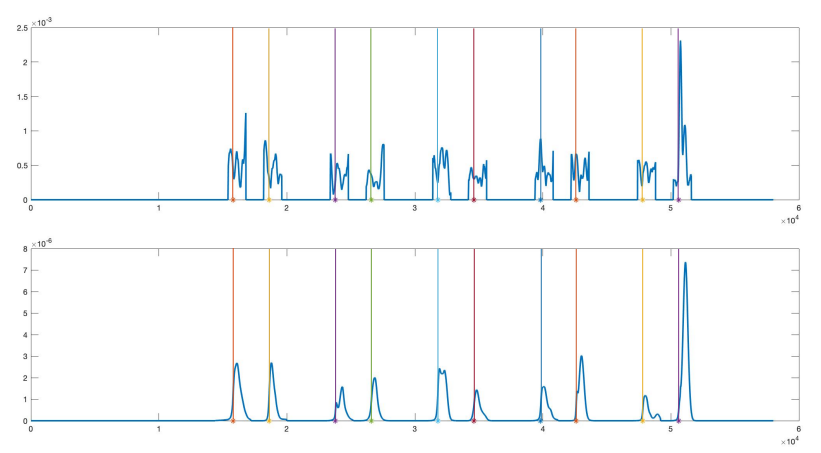

Figure 1. From the $L F / H F$ raw feature to the Autonomic Responsivity Function: exemplary estimates for one subject of (a) the $L F / H F$ raw feature properly derived, windowed and rescaled; (b) the Autonomic Responsivity Function $(A R F)$. Of note, the amplitude and latency of the autonomic response is persistent throughout the stimuli. Vertical lines corresponds to stimuli times.

and go back to zero level, we build the single response by modeling a gaussian function. The total responsivity curve is constructed from the extracted gaussian parameter. On a practical level, the corresponding curve has the latency of the peaks as mean and the width of peaks as standard deviation. Peaks width is rescaled by a factor of 0.7 in order to be consistent with the requirements of a gaussian distribution, in which $70 \%$ of observations fall in $\pm \sigma$ from mean. The maximum amplitude of the bell is then scaled by a factor equal to the maximum prominence of the bal peak. We proceed by substituting the gaussian responses within each response-window in a support function which has been previously filled with zero values outside each response-window. In this way the outcome function has zeros where no stimuli occur and has bell-shaped response curves where stimuli have been provided. Figure 1 shows the transformation from LF/HF peaks to ARF. Finally, areas and latency of ARF curves are computed from gaussian parameters. Stimulus-wise, the linear regression over areas (and latency) has been computed and the first order coefficient has been taken as habituation parameter. A composite habituation metrics is thus computed as:

$$
H A B(i)=\operatorname{atan}(a(i) \cdot l(i))
$$

where $H A B(i)$ is the habituation composite metrics for the $\mathrm{i}$-th subject, $a(i)$ is the slope coefficient of response areas of subject $\mathrm{i}$ and $l(i)$ is the slope coefficient of response latency of subject $l(i)$. When $H A B(i)$ is negative, we said that habituation has taken place; on contrary, a positive $H A B(i)$ value is informative of non habituation patterns, that is sensitization.

\section{Results}

The Short Sensory Profile (SSP-2) [12] and the Autism Diagnostic Observation Schedule (ADOS-2) [13] questionnaires have been exploited as gold standard for validating our metrics, particularly concerning the sensory profile delineation. With this aim in mind, we computed the Pearson's coefficient between $H A B$ and each questionnaire. We conducted a first simple correlation analysis correlating the composite habituation metrics with questionnaires and sensory profiles. Values show that the percentage representing the interaction between $H A B$ and the sensory profile of subjects (SSP-2) results is on average 59\%, whereas Pearson's coefficient between $H A B$ and Autism Diagnostic Observation Schedule (ADOS-2) is 0.76. Since SSP-2 assessment includes four areas of investigation, it is worth to mention the correlations of $H A B$ with each sensory response type: coefficients are $0.57,0.61,0.60$ and 0.56 respectively for registration, seeking, sensitivity, and avoiding sensory processing patterns. As literature considers values of correlation bigger or equal to 0.3 as relevant, we believe our result highlights how our metrics is a good estimation of the sensory profile. Such results help in targeting whether or not habituation metrics are representative of sensory profile which might be relevant as a robust proxy of sensory investigation, for instance in psychological or mental disorders. At this regard, Figure 2 graphically show the correlation presented. Of note, subjects' sensory scores align quite exquisitely with habituation metrics, suggesting the robustness of $H A B$.

\section{Conclusions}

In conclusion, our work has focused on defining habituation from dynamics estimated by a model of autonomic control to the heart. In particular we have devised a novel Autonomic Reactivity Function (ARF) via a point process model of heartbeat dynamics, describing the time-varying heart rate variability related to the Autonomic Nervous System (ANS) response . Based on ARF parameters, we have thus defined habituation metrics (HAB) directly correlated to sensory abnormalities. Finally, we have tested the mathematical formalization of such metrics in both neurotypical subjects and children with autism spectrum disorder. Results show a relevant positive correlation between HAB metrics and the patient psycho-sensory profiles, providing a first preliminary validation of the metrics.

\section{Acknowledgments}

This work was supported by Progetto Roberto Rocca, MIT-Italy Program and by a grant from the Simons Foundation (SFARI). We are grateful to the families who participated in our research for their time and dedication to the 

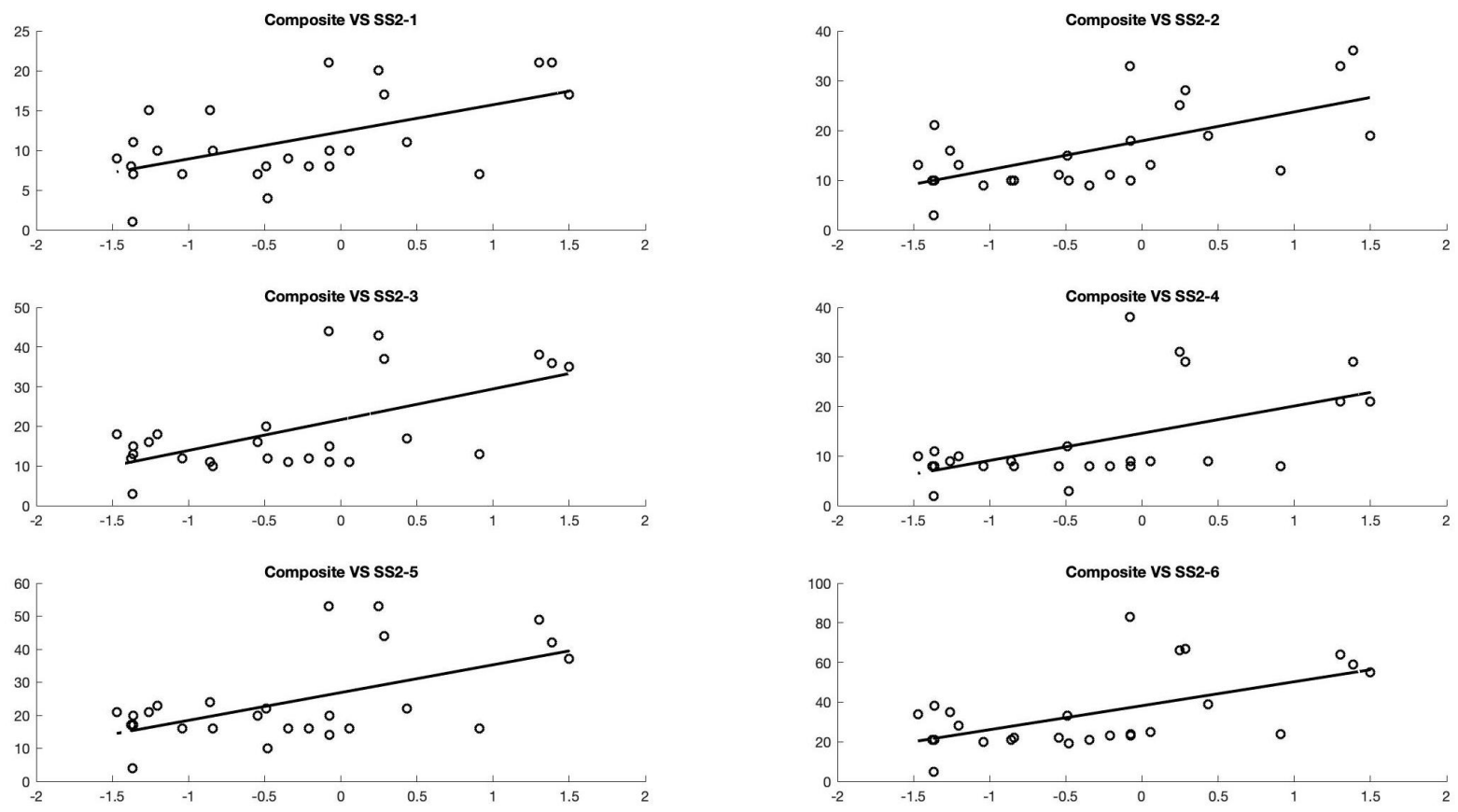

Figure 2. Relationship between HAB scores and sensory profile scores. The rate of habituation is predicted by the sensory scores of the SSP-2 across all subjects. Lines show linear fit, dots correspond to subjects.

pursuit of better understanding autism spectrum disorder.

\section{References}

[1] Guiraud JA, Kushnerenko E, Tomalski P, Davies K, Ribeiro $\mathrm{H}$, Johnson MH, Team B, et al. Differential habituation to repeated sounds in infants at high risk for autism. Neuroreport 2011;22(16):845-849.

[2] Engen T. Psychophysics. In States of brain and mind. Springer, 1988; 89-91.

[3] Martineau J, Roux S, Garreau B, Adrien J, Lelord G. Unimodal and crossmodal reactivity in autism: presence of auditory evoked responses and effect of the repetition of auditory stimuli. Biological psychiatry 1992;

[4] Perry W, Minassian A, Lopez B, Maron L, Lincoln A. Sensorimotor gating deficits in adults with autism. Biological psychiatry 2007;61(4):482-486.

[5] Lawson RP, Aylward J, White S, Rees G. A striking reduction of simple loudness adaptation in autism. Scientific reports 2015;5:16157.

[6] Barry RJ, James AL. Coding of stimulus parameters in autistic, retarded, and normal children: evidence for a two-factor theory of autism. International Journal of Psychophysiology 1988;6(2):139-149.

[7] Webb SJ, Jones EJ, Merkle K, Murias M, Greenson J, Richards T, Aylward E, Dawson G. Response to familiar faces, newly familiar faces, and novel faces as assessed by erps is intact in adults with autism spectrum disorders. International Journal of Psychophysiology 2010;77(2):106117.
[8] Dunn W. Infant/toddler sensory profile: user's manual. Pearson, 2002.

[9] Camm AJ, Malik M, Bigger JT, Breithardt G, Cerutti S, Cohen RJ, Coumel P, Fallen EL, Kennedy HL, Kleiger R, et al. Heart rate variability: standards of measurement, physiological interpretation and clinical use. task force of the european society of cardiology and the north american society of pacing and electrophysiology 1996;.

[10] Cacioppo JT, Tassinary LG, Berntson G. Handbook of psychophysiology. Cambridge University Press, 2007.

[11] Barbieri R, Matten EC, Alabi AA, Brown EN. A pointprocess model of human heartbeat intervals: new definitions of heart rate and heart rate variability. American Journal of Physiology Heart and Circulatory Physiology 2005; 288(1):H424-H435.

[12] Tomchek SD, Dunn W. Sensory processing in children with and without autism: a comparative study using the short sensory profile. American Journal of occupational therapy 2007;61(2):190-200.

[13] Hus V, Gotham K, Lord C. Standardizing ados domain scores: Separating severity of social affect and restricted and repetitive behaviors. Journal of autism and developmental disorders 2014;44(10):2400-2412.

Address for correspondence:

Lara Cavinato

Via Giuseppe Ponzio, 34, 20133 Milano, MI

lara.cavinato@mail.polimi.it 